\title{
A Verified Packrat Parser Interpreter for Parsing Expression Grammars ${ }^{*}$
}

\author{
Clement Blaudeau \\ Ecole Polytechnique \\ Palaiseau Cedex, France \\ clement.blaudeau@polytechnique.edu
}

\author{
Natarajan Shankar \\ Computer Science Laboratory \\ SRI International \\ Menlo Park, CA, USA \\ shankar@csl.sri.com
}

\begin{abstract}
Parsing expression grammars (PEGs) offer a natural opportunity for building verified parser interpreters based on higherorder parsing combinators. PEGs are expressive, unambiguous, and efficient to parse in a top-down recursive descent style. We use the rich type system of the PVS specification language and verification system to formalize the metatheory of PEGs and define a reference implementation of a recursive parser interpreter for PEGs. In order to ensure termination of parsing, we define a notion of a well-formed grammar. Rather than relying on an inductive definition of parsing, we use abstract syntax trees that represent the computational trace of the parser to provide an effective proof certificate for correct parsing and ensure that parsing properties including soundness and completeness are maintained. The correctness properties are embedded in the types of the operations so that the proofs can be easily constructed from local proof obligations. Building on the reference parser interpreter, we define a packrat parser interpreter as well as an extension that is capable of semantic interpretation. Both these parser interpreters are proved equivalent to the reference one. All of the parsers are executable. The proofs are formalized in mathematical terms so that similar parser interpreters can
\end{abstract}

\footnotetext{
${ }^{*}$ This work was supported by the National Institute of Aerospace Award C18-201097-SRI, NSF Grant SHF-1817204, Ecole Polytechnique, and DARPA under agreement number HR001119C0075. The views and conclusions contained herein are those of the authors and should not be interpreted as necessarily representing the official policies or endorsements, either expressed or implied, of NASA, NSF, DARPA, Ecole Polytechnique, or the U.S. Government. We thank the anonymous referees for their detailed comments and constructive feedback.
}

Permission to make digital or hard copies of all or part of this work for personal or classroom use is granted without fee provided that copies are not made or distributed for profit or commercial advantage and that copies bear this notice and the full citation on the first page. Copyrights for components of this work owned by others than the author(s) must be honored. Abstracting with credit is permitted. To copy otherwise, or republish, to post on servers or to redistribute to lists, requires prior specific permission and/or a fee. Request permissions from permissions@acm.org. CPP '20, January 20-21, 2020, New Orleans, LA, USA

( $) 2020$ Copyright held by the owner/author(s). Publication rights licensed to ACM.

ACM ISBN 978-1-4503-7097-4/20/01 ..\$15.00

https://doi.org/10.1145/3372885.3373836 be defined in any specification language with a type system similar to PVS.

CCS Concepts • Theory of computation $\rightarrow$ Grammars and context-free languages; Automated reasoning; • Software and its engineering $\rightarrow$ Syntax.

Keywords PVS, PEG grammar, packrat parsing, semantic parsing, verified parser, abstract syntax tree, well-formed grammars

\section{ACM Reference Format:}

Clement Blaudeau and Natarajan Shankar. 2020. A Verified Packrat Parser Interpreter for Parsing Expression Grammars. In Proceedings of the 9th ACM SIGPLAN International Conference on Certified Programs and Proofs (CPP '20), January 20-21, 2020, New Orleans, LA, USA. ACM, New York, NY, USA, 15 pages. https://doi.org/10. $1145 / 3372885.3373836$

\section{Introduction}

Parsing is the process of extracting structure and information from a string of tokens according to a formal grammar [1]. For critical applications, parsing errors and lack of proper input validation can be a common source of vulnerability and inconsistency leading to numerous errors and attacks [6]. Parser issues can come from the failure to follow a formal grammar, and from the formal grammar itself. Grammars might, by design or accident, introduce complexity, nontermination, and nondeterminism. For example, the dangling else problem where both if $A$ then $B$ and if $A$ then $B$ else $C$ are well-formed, leads to an ambiguity in parsing an expression of the form if $A$ then if $B$ then $C$ else $D$ where the else branch could be associated with either of the two conditionals. Ford [11] introduced Parsing Expression Grammars (PEGs) as a formalism for defining unambiguous grammars that can be parsed efficiently using a recursive descent scheme with parsing combinators for the basic grammar operators [7, 12, 13]. We present a PVS [21] formalization of PEGs with a rigorous treatment of well-formed grammars and a reference parser generator for such grammars. We also verify a packrat parser generator for PEGs and demonstrate that it is equivalent to the reference parser generator. Our parse tree representation can serve as an independent proofof-parse for PEGs making it possible to validate the result 
produced by the correct parsing, successful or unsuccessful, of an input string with reference to a given grammar regardless of how the parsing was performed. ${ }^{1}$

Parsing expression grammars were introduced as a pragmatic compromise between efficiency and expressiveness. These grammars are similar to context-free grammars in supporting terminal symbols, concatenation $A ; B$, and iteration $A^{*}$, but the choice operation $A \mid B$ is replaced with a priority operation $A / B$ where the grammar $B$ is matched against the input only when the match on $A$ fails. PEGs also include option (?), test (\&), and negation operations (!): ?A either matches (and consumes) some prefix of the current input or succeeds without consuming any tokens; $\& A$ tests if there is a prefix of the input that matches $A$ without consuming any tokens, and ! $A$ fails if some input prefix matches $A$. PEGs are unambiguous: there is at most one way to parse the input with respect to a given grammar. PEGs are greedy in that compared to the context-free analog, a PEG grammar represents the longest parse. For example, parsing an input with respect to $A^{*}$ will consume as many instances of $A$ from the input as possible. The test operations can be used to define test predicates that can look ahead into arbitrarily long prefixes of the input without actually consuming any input, making it possible to even capture certain non-context-free grammars such as $a^{n} b^{n} c^{n}$.

A PEG grammar consists of a set of productions mapping nonterminals to PEG expressions in the nonterminals. Not all PEGs are well-formed. For example, $A^{*}$ is not wellformed when $A$ can match the empty string. A left-recursive grammar like $A \rightarrow A / a$ is not well-formed since the corresponding unfolding of the grammar might not converge.

The parsing of well-formed PEGs can be directly implemented by a recursive descent parser in which each construct is supported by a parsing combinator. For example, the choice construct $A / B$ is implemented by a combinator that takes a parser for $A$ and a parser for $B$, and invokes the parser for $B$ on the input only when the parser for $A$ returns with failure. This kind of parsing scheme can have exponential complexity through repeated parsing queries with the same nonterminal. Consider the following grammar [10]:

$$
\begin{aligned}
A & := \\
M & ::=G+A / M \\
G & ::=(A) / \text { int }
\end{aligned}
$$

Parsing an input, say $(((5 * 4) * 3) * 2) * 1$ requires $2^{4}$ parser calls for the nonterminal $M$. This duplication is avoided in packrat parsing by memoizing the results of the parse.

\footnotetext{
${ }^{1}$ The PVS formalizations can be accessed at https://github.com/SRI-CSL/ PVSPackrat.
}

PEGs and PEG parsing is defined in Section 2 where we also discuss the termination problem for parsing with respect to arbitrary PEGs. In Section 3, we outline the wellformedness properties of PEGs that guarantee the termination of parsing. We present the verification of three parser interpreters for PEGs. The first is a reference parser interpreter for PEG grammars presented in Section 5. The second is a packrat parser interpreter described in Section 6. Section 7 describes a variant that allows the output parse tree to be customized according to semantic actions. Section 8 makes some brief concluding observations.

Related Work. Though parsing is an important firewall between untrusted data and vulnerable applications, there have only been a few instances of verified parsers or parser interpreters/generators. The parser front-end was one of the few identifiable weaknesses of the verified CompCert compiler [18]. Barthwal and Norrish [2] present an SLR parser generator verified in HOL4 that produces an independently verifiable parse tree. Ridge [23] has verified the termination, soundness, and completeness of a recursive descent parser based on parsing combinators for context-free languages. The RockSalt checker of Morrisett, Tan, Tassarotti, Tristan, and Gan [20] for checking software-based fault isolation of native executable code in a browser employs a regular expression parser for $\mathrm{x} 86$ instructions that has been verified to be sound in Coq. In subsequent work, Tan and Morrisett [24] certified encoder/decoder pairs are constructed from bidirectional grammars. Lopes, Ribeiro, and Camarão [19] have also verified a regular expression parser using Idris [5]. Bernardy and Jansson [3] have formalized Valiant's algorithm for parsing context-free languages in Agda [4]. Koprowski and Binsztok [15] present a Coq verification of a parser interpreter called TRX for PEGs. The CakeML compiler which has been verified using HOL4 employs a similar verified PEG parser interpreter [16]. Lasser, Casinghino, Fisher, and Roux [17] have verified an LL(1) parser generator covering the generation of the lookup table and the stack-based parser. Jourdan, Pottier, and Leroy [14] define a generator for an independently and verifiably validatable parsing automaton for an LR(1) grammar. A number of papers address the verification and synthesis of decoder/encoder pairs [8, 22, 25-27] where the objective is to serialize and deserialize data.

Of the related projects, the TRX parser [15] and the CakeML parser [16] are closest to the one presented here. We build on the TRX work, particularly in the treatment of termination for PEGs and in the definition of the non-packrat PEG parser. However, we define an executable check for grammar well-formedness in contrast to the inductive characterization in TRX. We also present a parse tree representation of both successful and failed parses. We show that these parses are unique for a given grammar thus capturing both the soundness and completeness of the parser interpreter. The TRX verification only captures the soundness argument 
using an inductively defined parse derivation that captures the parse semantics. While parse trees have been used as proofs-of-parse, in the work of Barthwal and Norrish [2] and Ridge [23], we have extended it to capture both success and failure. We also go beyond the reference PEG parser interpreter to verify two packrat parser interpreters, one without semantic actions and one with. The PVS proofs we present take advantage of predicate subtyping in PVS to craft a verification methodology that reduces the correctness of the grammar analyzer and the parser interpreters to small, local proof obligations with easy automated proofs instead of big theorems with manually generated proof structures. Our proof methodology is transferable to other parsing formats and parser generation algorithms.

\section{Parsing Expression Grammars}

Parsing Expression Grammars (PEGs) were introduced by Ford as a formalism for capturing grammars that correspond to greedy, unambiguous recursive descent parsing. To describe PEGs, we rely on operators resembling those in context-free grammars, with the notable exception of the prioritized choice operator. Here is a formal definition of PEGs, relying on two types: $V_{T}$, the type of terminals (in most cases bytes or characters, but applicable to any type instantiating $V_{T}$ ) and $V_{N}$, the type of nonterminals that are basically patterns: the set of grammar expressions $\Delta$ is inductively defined as below.

$\begin{array}{cccl}\Delta::= & \epsilon & \text { empty expression } & \\ & {[\cdot]} & \text { any character } & \\ & {[a]} & \text { a terminal } & \left(a \in V_{T}\right) \\ & A & \text { a nonterminal } & \left(A \in V_{N}\right) \\ & e 1 ; e 2 & \text { a sequence } & (e 1, e 2 \in \Delta) \\ e 1 / e 2 & \text { a prioritized choice } & (e 1, e 2 \in \Delta) \\ \mid & e * & \text { a greedy repetition } & (e \in \Delta) \\ \mid e & \text { a not-predicate } & (e \in \Delta)\end{array}$

In addition to those basic operators, we have a few other operators that can be emulated by the basic ones:

1. $[a-z]$ the range, equivalent to $[a] /[b] / \ldots /[z]$

2. [" $s$ "] the string, equivalent to $\left[c_{1}\right] ;\left[c_{2}\right] ; \ldots ;\left[c_{n}\right]$, where $s=c_{1} c_{2} \ldots c_{n}$ for tokens $c_{1}, \ldots, c_{n}$

3. $e+$ the plus operator, equivalent to $e ; e *$

4. $e$ ? the optional operator, equivalent to $e / \epsilon$

5. \&e the and operator, equivalent to !!e

In practice these additional operators would certainly be used, but as they can be emulated by the basic ones, we can ignore them for the proofs in order to avoid redundant cases during the case analyses.

Restriction to a Finite Set of Nonterminals. Technically we could consider $V_{N}$ as any set, finite or not, and build results making no other assumptions. In practice however, $V_{N}$ is the set of patterns given by the creator of the grammar. So

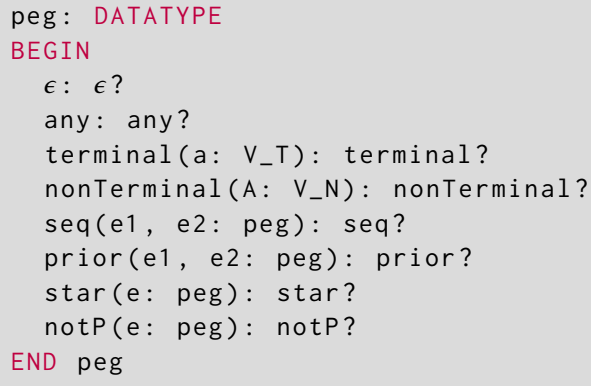

Figure 1. PVS code for the PEG grammars formalized as an algebraic datatype with constructors, accessors, and recognizers

we can consider $V_{N}$ finite and bounded by $n+1=\operatorname{Card}\left(V_{N}\right)$. In the following, $V_{N}$ and $\llbracket 0, n \rrbracket$ are used interchangeably.

The Problem of Termination. Though PEGs are unambiguous [11], this does not ensure that parsing terminates. The most basic non-terminating PEG expression is $\epsilon *$ since parsing with it loops forever without consuming any input. It shows that greedy operators should not rely on expressions that can succeed without consuming tokens from the input. Also, the use of nonterminals can easily introduce nonterminating left-recursion. For example, with $V_{N}=\{A, B\}$ and grammar production map $P_{\text {exp }}$ from $V_{N}$ to $\Delta$ defined as:

$$
\begin{aligned}
& P_{\text {exp }}(A)=B \\
& P_{\text {exp }}(B)=A
\end{aligned}
$$

The parsing here would loop forever. This introduces the two main considerations regarding the termination of parsing: consumption of characters and prevention of infinite leftrecursion. In the following section, we define the properties of a well-formed grammar that ensure termination of parsing regardless of the input.

\section{Well-Formed PEG Grammars}

We develop a new approach to well-formed PEGs with a computational point of view. Our focus is on constructive definitions that yield easy implementations. The notions of structural well-formedness and pattern well-formedness are, along with the use of abstract proof-of-parse trees, the major differences with respect to the verified PEG parser TRX [15].

\subsection{Consumption and Grammar Properties}

To characterize terminating grammars, we first need to identify the relevant properties of grammar expressions that ensure termination. We then need to compute them. The most basic property is success without consumption (of tokens). However, the not-predicate, which succeeds if the expression fails, requires us to also know if expressions can fail. The last case is success with consumption of at least one 
token of the input. We introduce below a precise mathematical formalization of the notion of a grammar property that corresponds to the way it is designed and proved in PVS.

Formalization. To translate those properties, we define three predicates:

$$
P_{\perp}, P_{0}, P_{>0}: \Delta \longrightarrow \text { bool, }
$$

representing whether a parse based on the grammar can fail, succeed without consuming input, or succeed only by consuming input, respectively. We define $P_{\geq 0}(e)$ as $P_{0}(e) \vee$ $P_{>0}(e)$. The inductive definition relies on the rules shown in Figure 2.

This inductive approach is valid and matches the intuition, and can be easily formalized in PVS. However, we want an effective computational mechanism for checking the tree structure of a grammar expression that might contain loops ${ }^{2}$. We can tackle this problem as follows: we first introduce the set of already known properties of nonterminals, that starts as the empty set. Then we try to compute all the new properties that can be obtained with current knowledge, and iterate until no new properties are found. The number of computed properties of nonterminals is nondecreasing, and bounded by $3 \times\left|V_{N}\right|$. When all the properties of each nonterminal are known, we can compute in a straightforward recursive way the properties of any grammar involving these nonterminals. We formalize this approach below by first introducing a function over the nonterminals: $P: V_{N} \longrightarrow\{\text { known, unknown }\}^{3}$ that represent the known properties. Let $\mathbb{P}$ be the set of those predicates. In the actual implementation, we use bool for representation of known/unknown. It is important to notice that in our model, a false does not mean that the property is false, but that it is unknown, so that only true yields useful information. We introduce an order:

$$
\forall P, P^{\prime} \in \mathbb{P}, P \leq P^{\prime} \Rightarrow \forall A \in V_{N}\left\{\begin{array}{l}
P(A)(1) \Rightarrow P^{\prime}(A)(1) \\
P(A)(2) \Rightarrow P^{\prime}(A)(2) \\
P(A)(3) \Rightarrow P^{\prime}(A)(3)
\end{array}\right.
$$

This order is trivially reflexive and transitive. We can now introduce a function that computes the properties of a grammar node based on current knowledge: the code is given in Figure 3.

$$
g: \Delta \times \mathbb{P} \longrightarrow[\text { bool }]^{3}
$$

where

$$
\begin{aligned}
P_{\perp}: \Delta \longrightarrow \text { bool } & =g(\cdot, P)(1) \\
P_{0}: \Delta \longrightarrow \text { bool } & =g(\cdot, P)(2) \\
P_{>0}: \Delta \longrightarrow \text { bool } & =g(\cdot, P)(3)
\end{aligned}
$$

This structurally recursive function satisfies the rules from Figure 2. The termination measure pegMeasure $(G)$ is just the size of $\mathrm{G}$ given by the number of nodes. We also introduce a function $\rho$ that takes a nonterminal $A$ and a set of properties

\footnotetext{
${ }^{2}$ With $A$ relying on the properties of $B$ and vice-versa
}

$P$, compute the properties of $A$ and returns $P$ extended with the new computed properties:

$$
\begin{aligned}
\rho: V_{N} \times \mathbb{P} & \longrightarrow \mathbb{P} \\
(A, P) & \longmapsto\left\{\begin{array}{l}
\rho(A, P)(A)=g\left(P_{\exp }(A), P\right) \\
\rho(A, P)(B)=P(B)(B \neq A)
\end{array}\right.
\end{aligned}
$$

Basically, we want to apply this function $\rho$ a certain number of times (at most $3 \times\left|V_{N}\right|$ ) to get all reachable properties. But the problem is that this function is not monotonic, for example, when $P(A)=\left(\right.$ known, known, known) and $P_{\text {exp }}(A)=\epsilon$, $\rho(A, P)<P$. Thus, we need to restrain the set of properties to what we call coherent properties: sets of properties that are not contradicted by themselves. We define that set as:

$$
\mathbb{C}=\left\{P \in \mathbb{P} \mid \forall A \in V_{N}, P \leq \rho(A, P)\right\}
$$

Under this assumption of coherence, $\rho$ is monotonic.

Lemma 3.1 ( $\rho$ is monotonic).

$$
\forall P, P^{\prime}, G \in \mathbb{C}^{2} \times \Delta, P \leq P^{\prime} \Rightarrow \rho(G, P) \leq \rho\left(G, P^{\prime}\right)
$$

In the PVS implementation, we define the $\rho$ function, as well as a coherent properties type, and prove the monotonicity result.

\subsection{Properties Computation}

Now that we have a clear formalization of the computation of grammar properties, we can focus on the way all the properties are recursively computed. Again, the main idea is that we only need the properties of the nonterminals to compute the properties of any grammar node with a simple recursive function.

Example. Consider the example with the following $P_{\exp }$ function:

$$
\begin{aligned}
& P_{\text {exp }}(A)=[a] \\
& P_{\exp }(B)=! A / C \\
& P_{\exp }(C)=! B ; A
\end{aligned}
$$

1. We have $P_{\perp}(A)$ and $P_{>0}(A)$ immediately.

2. We then get $P_{\perp}(! A)$ and $P_{0}(! A) \Rightarrow P_{0}(B)$.

3. This gives us $P_{\perp}(! B) \Rightarrow P_{\perp}(C)$.

4. Combining $P_{\perp}(! A)$ and $P_{\perp}(C)$ we get $P_{\perp}(B)$.

In this example we can see that for computing the properties of $B$ we need properties of $C$ and vice-versa: the computation cannot be done in a single pass.

Computation Process. The computation process of the nonterminal properties is the following: ${ }^{3}$

1. Starting with the empty set of properties $0_{\mathbb{C}}$, we compute the properties of all the nonterminals, one by

\footnotetext{
${ }^{3}$ It is interesting to notice that far more optimal ways could be invented to tackle this computational problem, especially using graphs and memoization to prevent computing over and over again the same things. But first of all, the aim here is to have a verified solution, and secondly, this computation is only done once and for all, it does not affect the parsing. Thus the optimality is not the main focus here.
} 


$$
\begin{aligned}
& \overline{P_{0}(\epsilon)} \quad \overline{P_{>0}([\cdot])} \quad \overline{P_{\perp}([\cdot])} \quad \frac{a \in V_{T}}{P_{>0}([a])} \quad \frac{a \in V_{T}}{P_{\perp}([a])} \quad \frac{P_{\perp}(e)}{P_{0}(e *)} \quad \frac{P_{>0}(e)}{P_{>0}(e *)} \\
& \begin{array}{cccc}
\star \in\{0,>0, \perp\} & A \in V_{N} & P_{\star}\left(P_{\exp }(A)\right) & \frac{P_{\perp}\left(e_{1}\right) \vee\left[P_{\geq 0}\left(e_{1}\right) \wedge P_{\perp}\left(e_{2}\right)\right]}{P_{\star}(A)}
\end{array} \\
& \frac{P_{0}\left(e_{1}\right) \quad P_{0}\left(e_{2}\right)}{P_{0}\left(e_{1} ; e_{2}\right)} \quad \frac{\left[P_{>0}\left(e_{1}\right) \wedge P_{\geq 0}\left(e_{2}\right)\right] \vee\left[P_{0}\left(e_{1}\right) \wedge P_{>0}\left(e_{2}\right)\right]}{P_{>0}\left(e_{1} ; e_{2}\right)} \\
& \frac{P_{0}\left(e_{1}\right) \vee\left[P_{\perp}\left(e_{1}\right) \wedge P_{0}\left(e_{2}\right)\right]}{P_{0}\left(e_{1} / e_{2}\right)} \quad \frac{P_{>0}\left(e_{1}\right) \vee\left[P_{\perp}\left(e_{1}\right) \wedge P_{>0}\left(e_{2}\right)\right]}{P_{>0}\left(e_{1} / e_{2}\right)} \\
& \frac{P_{\perp}\left(e_{1}\right) \quad P_{\perp}\left(e_{2}\right)}{P_{\perp}\left(e_{1} / e_{2}\right)} \quad \frac{P_{\perp}(e)}{P_{0}(! e)} \quad \frac{P_{\geq 0}(e)}{P_{\perp}(! e)}
\end{aligned}
$$

Figure 2. Inductive rules for grammar properties

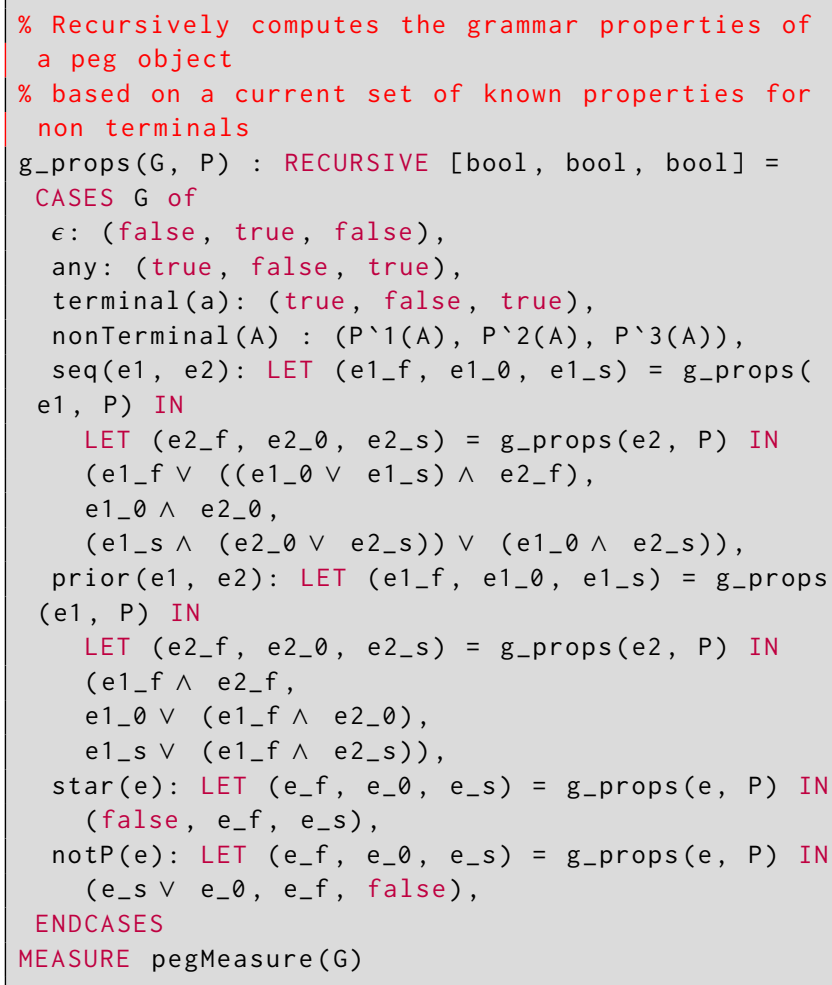

Figure 3. PVS implementation of the $g$ function defined by case analysis. PVS allows the use of the unicode characters for and, or, implies, iff.

one, augmenting the set of properties as new ones are found.

2. Once that is done, we check if new properties have been found since the start of the nonterminal computation. If so, we restart the computation, and otherwise, we return the result.
Formalization. Next, we formalize the computation process. We define a sequence that translates the computation of properties for all the nonterminals, one by one. The property set on which the computation is made is the superscript, and the nonterminal on which we are trying to compute new properties is written in subscript. So to recompute the new properties for all nonterminals between 0 and $n$ we have:

$$
\left\{\begin{array}{l}
r_{A}^{P}=r_{A+1}^{\rho(A, P)} \\
r_{n}^{P}=\rho(n, P)
\end{array}\right.
$$

Lemma 3.2 captures three useful properties entailed by this sequence.

Lemma 3.2 (Recomputing nonTerminal properties increases knowledge).

$$
\begin{array}{rrr}
\forall P, A \in \mathbb{C} \times \llbracket 0, n \rrbracket, & P & \leq r_{A}^{P} \\
\forall P, P^{\prime}, A \in \mathbb{C}^{2} \times \llbracket 0, n \rrbracket, & P \leq P^{\prime} \Rightarrow r_{A}^{P} & \leq r_{A}^{P^{\prime}} \\
\forall P, A \in \mathbb{C} \times \llbracket 0, n-1 \rrbracket, & r_{A+1}^{P} & \leq r_{A}^{P}
\end{array}
$$

The expected result of the computation is a set of properties that cannot be extended (because it already has all the reachable properties). We can define the set of properties that are such fixpoints of computation:

$$
\mathbb{F}=\left\{P \in \mathbb{C} \mid P=r_{0}^{P}\right\}
$$

Such a fixpoint can be reached in a bounded (by $3 *(n+1)$ ) number of steps by repeatedly applying the function $\phi(P)=$ $r_{0}^{P}$ starting with $0_{\mathbb{C}}$. We have the following results:

Theorem 3.3 (Fixpoint properties). Recomputing the properties of a nonterminal with a set of properties that is a fixpoint gives back the same result:

$$
\forall(P, A) \in \mathbb{F} \times V_{N}, P=\rho(A, P)
$$

In the PVS implementation, we define the $r$ function, as well as a fixpoint properties type. We prove the lemmas and the theorem. This gives us an effective way to compute the properties of a given set of nonterminals. 


\subsection{Well-Formed Grammars}

Those properties allow us to define the grammar that we call well-formed: grammars that structurally enforce the termination of the parsing. The main idea is to prevent the two kinds of loops that we mentioned: structural ones $(\epsilon *)$ and pattern ones $\left(P_{\text {exp }}(A)=B, P_{\text {exp }}(B)=A\right)$. Two approaches are needed:

1. Preventing structural aberrations is quite easy: we can just go through the whole tree and check that every time the star operator is used, it is applied to a grammar node that cannot succeed without consuming any input. Thanks to the work previously done on properties computation, this is an easy task.

2. Preventing pattern aberrations is a bit trickier, as we want to allow patterns to use other patterns in some instances while preventing them from doing so in others. The idea is the following: we assume there exists an order ${ }^{45}$ over the nonterminals where the grammar $P_{\text {exp }}(A)$ for $A$ can only employ strictly smaller nonterminals until it is clear that at least one character is consumed. For example, once the left-branch of a seq is not of type $P_{0}$, the right branch can use any nonterminal.

A visual representation is given in Figure 4.

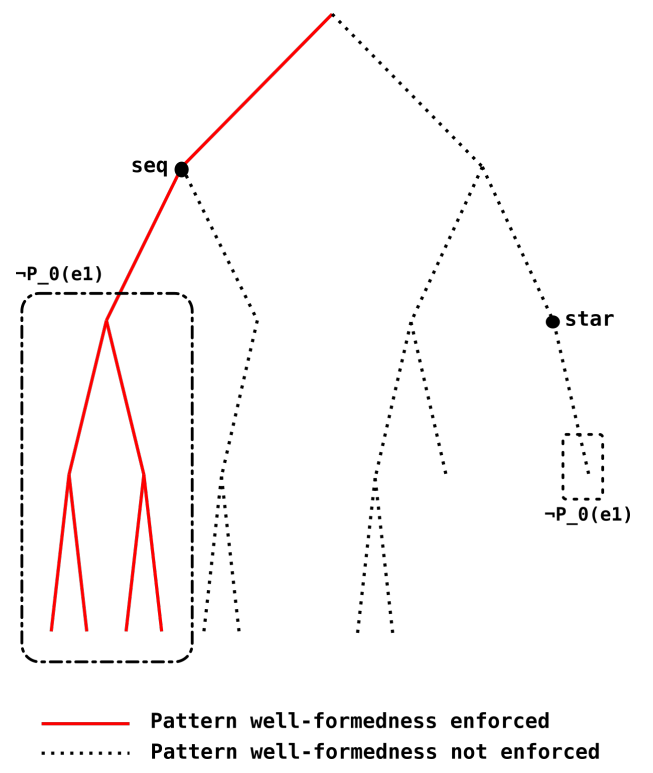

Figure 4. Representation of a well-formed grammar

\footnotetext{
${ }^{4}$ This approach is actually equivalent to the inductive definition of wellformedness that can be found in [10]: if we have an order on nonterminals then the inductive definition can follow that order, and vice-versa

${ }^{5}$ For the scope of this paper, we assume that the user is able to provide the order for the nonterminals. We conjecture that if such an order actually exists, there exists ways to compute it that are more efficient than testing all possible orders. An approach using a graph of dependency between the nonterminals might be fruitful.
}

Formalization. Given those remarks we see that we can define a function $\omega$ that verifies both structural and pattern well-formedness of a grammar node that is a subterm of $P_{\exp }(A)$ for a certain $A \in V_{N}$. The function always checks structural well-formedness, but pattern well-formedness is only checked on certain branches, using a special argument $\delta$ that is true if we enforce pattern well-formedness and that is false if it is not needed.

$$
\begin{aligned}
& \omega: \Delta \times V_{N} \times \text { bool } \longrightarrow \text { bool } \\
&(\epsilon, A, \delta) \longmapsto \top \\
&([\cdot], A, \delta) \longmapsto \top \\
&([a], A, \delta) \longmapsto \mathrm{\top} \\
&(B, A, \delta)\left.\longmapsto \delta \Rightarrow(B\rangle_{N} A\right) \\
&\left(e_{1} ; e_{2}, A, \delta\right) \longmapsto \omega\left(e_{1}, A, \delta\right) \\
&\left(e_{1} / e_{2}, A, \delta\right) \longmapsto \wedge\left(e_{2}, A, \delta \wedge P_{0}\left(e_{1}\right)\right) \\
&(e *, A, \delta) \longmapsto \omega\left(e_{1}, A, \delta\right) \wedge \omega\left(e_{2}, A, \delta\right) \\
&(! e, A, \delta) \longmapsto \omega(e, A, \delta) \wedge\left(\neg P_{0}(e)\right) \\
&
\end{aligned}
$$

The seq case is the most interesting: the new value for $\delta$ on the right branch is $\delta \wedge P_{0}\left(e_{1}\right)$. We check for pattern wellformedness in the right branch only if we are supposed to do so ( $\delta$ is true) and if the left branch can succeed without consuming any input $\left(P_{0}\left(e_{1}\right)\right)$.

Definition 3.4 (Well-formed grammars). We can define a well-formed set of grammars $P_{\exp }$ as satisfying:

$$
\forall A \in V_{N}, \omega\left(P_{\exp }(A), A, \top\right)
$$

Such grammars ensure termination of the corresponding parser.

In the PVS implementation, we define the $\omega$ function (named g_wf), as well as a well-formed set of nonterminals type.

\section{Abstract Syntax Trees and Parsing}

In contrast with prior work $[11,15]$, we choose not to define parsing as a relationship between an expression, a string and a result, but as a step of a parsing function that corresponds to the actual computation and that verifies a certain number of properties. To do so, we choose to define an output type that represents a computational path, that we call an $a b$ stract syntax tree as a parse tree that represent the full trace of the parse covering both successful and failed branches. This representation of the computational path as an explicit proof of correctness (soundness and completeness) for our reference parser, makes it easier to observe and explain both success and failure. Unlike the TRX verified PEG parser [15], we choose to use this structure and to avoid defining parsing through rules. We believe that this approach is richer, as it provides explicit information on the computational path, and as it is easy to show that the rules of parsing are verified 
at each node of an Ast tree. An example of a lemma capturing a parsing rule that is verified by the parser is shown in Figure 8 .

\subsection{Pre-Ast}

The implementation of those abstract syntax trees will need to verify a certain number of properties in order to represent an actual computational path. But as we can define properties only on existing objects, we start by creating a pre-Ast datatype, on which we will build the full Ast type. As we can expect, a pre-Ast depends on the type of terminals $V_{T}$, the type of non terminals $V_{N}$, but also the upper bound ${ }^{6}$ of the input $b \in \mathbb{N}$. We have nine cases, that corresponds to nine constructors. Each constructor has its own arguments, but always requires $s, e \in \llbracket 0, b \rrbracket$ : the start and end of the string that was consumed by the subtree.

1. $\operatorname{skip}(s, e, G)$ with $G \in \Delta$. This corresponds to all the cases where part of the grammar is skipped. For example, in the case of a prioritized choice, the second branch can be skipped if the first branch is already a success. For the moment, no condition is added on $s$ and $e$, but later on we will obviously ask for $e=s$. We give the set of skips: $\mathcal{S}=\{T \in \mathcal{A} \mid \exists(s, e, G) \in$ $\mathbb{N} \times \mathbb{N} \times \Delta, T=\operatorname{skip}(s, e, G)\}$.

2. $\epsilon(s, e)$ for the corresponding grammar node

3. $\operatorname{any}(s, e, x)$ for the corresponding grammar node, the the consumed character stored as $x$.

4. terminal $(s, e, a, x)$ for the corresponding grammar node, the expected character being $a$ and the consumed one being $x$. In the success case, $a=x$ but in the failure case, we want to store exactly why it failed, so we store $x \neq a$.

5. nonTerminal $(s, e, A, T)$ for the corresponding grammar node, with $T \notin \mathcal{S}$ being the tree for parsing the non terminal $A$.

6. $\operatorname{seq}\left(s, e, T_{1}, T_{2}\right)$ with $T_{1} \notin \mathcal{S}$. $T_{1}$ and $T_{2}$ are supposed to correspond to the parsing of the sub-expressions $e_{1}$ and $e_{2}$.

7. $\operatorname{prior}\left(s, e, T_{1}, T_{2}\right)$ with $T_{1} \notin \mathcal{S}$. Same as seq.

8. $\operatorname{star}\left(e, s, T_{0}, T_{s}\right)$ with $T_{0} \notin \mathcal{S}$. To keep the star structure, we ask for $T_{s}$ to be either of type star or skip. We define $\mathfrak{S}$ as the set of star-like pre-Ast.

9. $\operatorname{not} P(e, s, T)$ with $T \notin \mathcal{S}$.

Here is the summary:

\footnotetext{
${ }^{6}$ This can easily be replaced by a notion of end of stream character if the input bound is unknown.
}

Definition 4.1 (Pre-Ast). We give the following inductive definition:

$$
\begin{aligned}
& \mathcal{A}\left[V_{T}, V_{N}, b\right] \quad::=\operatorname{skip}(s, e, G),(G \in \Delta) \\
& \epsilon(s, e) \\
& \operatorname{any}(s, e, x),\left(x \in V_{T}\right) \\
& \text { terminal }(s, e, a, x),\left(a, x \in V_{T}\right) \\
& \text { nonTerminal }(s, e, A, T),\left(A \in V_{N}\right) \\
& \operatorname{seq}\left(s, e, T_{1}, T_{2}\right),\left(T_{1} \notin \mathcal{S}\right) \\
& \operatorname{prior}\left(s, e, T_{1}, T_{2}\right),\left(T_{1} \notin \mathcal{S}\right) \\
& \operatorname{star}\left(s, e, T_{0}, T_{s}\right),\left(\begin{array}{l}
T_{0} \notin \mathcal{S}, \\
T_{s} \in \mathcal{S} \cup \mathfrak{S}
\end{array}\right) \\
& \text { | } \operatorname{not} P(s, e, T),(T \notin \mathcal{S})
\end{aligned}
$$

where, $s, e \in \llbracket 0, b \rrbracket$

As we define the pre-Ast, we see that we want to add extra-conditions on the arguments of the constructors. But some of those conditions rely on a notion of failure/success, that we will formalize now.

\subsection{Failure and Success of Pre-Ast}

Computing the failure or success requires a depth-first traversal of the tree. We consider three possible outcomes: $\{\perp, \top, u\}$ with $u$ standing for undefined and we call this the type of a pre-Ast. Here is a description of the function $\eta$ that computes the type:

1. skip is always undefined: the failure/success should not depend on a skip.

2. $\epsilon$ is a success if $e=s$ (meaning nothing was consumed). Otherwise it is undefined.

3. any is a success if $e=s+1$, a failure if $e=s$ (meaning the end of string was reached and no character was consumed) or undefined otherwise.

4. terminal has the same conditions as any and adds the $a=x$ condition.

5. nonTerminal is of the same type as its subtree

6. seq is a success if both subtrees are successes, and a failure if $T_{1}$ fails or if $T_{1}$ succeeds and $T_{2}$ fails. All other cases are undefined.

7. prior is a success if $T_{1}$ is, or if $T_{1}$ fails and $T_{2}$ succeeds. If $T_{1}$ and $T_{2}$ fails, it is a failure, and undefined otherwise.

8. star is always a success, as soon as $T_{0}$ is not undefined. If $T_{0}$ is a success, $T_{s}$ must be a success too (and thus is not a skip) $)^{7}$.

9. not $P$ is the opposite of the subtree type, where the opposite of undefined is also undefined.

Definition 4.2 (Meaningful tree). A meaningful tree is a pre-Ast tree that is either of type $\perp$ or $\mathrm{T}$. If we write $\mathcal{M}$ the set of meaningful trees, we have:

$$
\mathcal{M}=\eta^{-1}(\{\perp, \top\})
$$

\footnotetext{
${ }^{7}$ This corresponds to the fact that until the search for a pattern $e$ fails, we
} keep on searching (the star is greedy). 


\subsection{Well-Formed Tree}

Once we have a standalone notion of failure and success, we can add the other conditions to make sure the tree is well-formed. Well-formed trees are basically the ones corresponding to a real computational path. Here is a summary of those conditions:

1. $\epsilon$, any, terminal are well-formed if they are meaningful.

2. nonterminal is well-formed if its subtree is and their bounds are equals: $e=e_{T}$ and $s=s_{T}$

3. seq: We require $T_{1}$ to be well-formed, and the bounds of $T_{1}$ and $T_{2}$ to be a partition of the bounds of $T: s=s_{1}$, $e_{1}=s_{2}$ and $e_{2}=e$. If $T_{1}$ is a failure, the second part of the grammar is not visited, so $T_{2}$ must be a skip $\left(T_{2} \in \mathcal{S}\right)$ and it should not consume anything $\left(s_{2}=e_{2}\right)$.

4. prior: We require $T_{1}$ to be well-formed, and both $T_{1}$ and $T_{2}$ should start at $s\left(s=s_{1}\right.$ and $\left.s=s_{2}\right)$. If $T_{1}$ is a success, then $T_{2}$ must be a non-consuming skip, and the end must be the one of $T_{1}\left(e=e_{1}\right)$. If $T_{1}$ is a failure, the end bound must be the one of $T_{2}$, that is not allowed to be a skip $\left(e=e_{2}\right.$ and $\left.T \notin \mathcal{S}\right)$

5. star: We require $T_{0}$ to be well-formed, and the bounds should be a partition (like with seq). If $T_{0}$ is a success, $T_{s}$ must be a star, and if $T_{0}$ is a failure, $T_{s}$ must be a non-consuming skip.

6. not $P$ is well-formed if its subtree is. It should not consume anything, so the bounds should be equal: $s=e$.

7. skip is always well-formed.

We write the set of well-formed trees $\mathcal{W}$. We have the following result.

Theorem 4.3 (Well-formed trees are meaningful). A wellformed tree is either a tree of success or of failure:

$$
\mathcal{W} \subset \mathcal{M}
$$

In PVS, we define the astType? and astWellformed functions over the pre_ast type. We prove the theorem by induction.

\subsection{The Definition of Parsing}

Usually, as we mentioned and as in [11] and [15], the parsing is defined as a relationship between the inputs and the outputs that verifies a certain number of derivation rules corresponding to all the parsing cases. Lemmas that link the properties of the grammar to the parsing relationship ${ }^{8}$ are then proved to ensure that any algorithm following the parsing rules would terminate. We chose not to follow this approach, but to include all the conditions and the lemmas in the typing system of PVS. This has several advantages:

\footnotetext{
${ }^{8}$ Those lemmas are the following : (1) If the parsing fails, the grammar is of type $P_{\perp}$ (2) If the parsing succeed without consuming any input, the grammar is of type $P_{0}$ (3) If the parsing succeed consuming at least one token, the grammar is of type $P_{>0}$.
}

- The typing system is verified by PVS, but once it is proven, it has no impact on the real computation. ${ }^{9}$

- We can rely on fewer axioms, as there is no need for a parsing relation-ship to be defined. All the axioms of the parsing relation-ship are proven properties of the parser, and such proofs are easy to do.

To prove that well-formed trees actually correspond to parsing of a given input given a grammar, we introduce two notions. We say that a well-formed tree $T$ is true to a grammar $G^{10}$ if we can rebuild $G$ from $T$. We say that a well-formed tree $T$ is true to an input $I$ if the characters stored in the tree at some starting indices corresponds to the input. This notion only applies to the part of the input covered by the tree namely, between the start and the end indices of the tree ${ }^{11}$. This yields three results that can be proved by induction (see Figure 5 for the PVS code):

Theorem 4.4 (Uniqueness for well-formed trees). We have:

- If a grammar $G_{1}$ and a grammar $G_{2}$ are both true to a given well-formed tree $T$, then $G_{1}=G_{2}$.

- If an input $I_{1}$ and an input $I_{2}$ are both true to a given well-formed tree $T$, and if the starting and ending indexes of the tree are $(s, e)$, then $\forall i \in \llbracket s, e \rrbracket, I_{1}(i)=I_{2}(i)$

- If two well-formed trees $T_{1}$ and $T_{2}$ are both true to the same grammar $G$ and to the same input $I$, and start at the same point $\left(s_{T_{1}}=s_{T_{2}}\right)$, then $T_{1}=T_{2}$

This result is needed to prove that a parser is complete. Since the parser produces well-formed trees that are true to the grammar and input given as arguments, we get that the result tree is the only one possible. If we add the fact that well-formed trees are always either trees of success or of failure, we get that parsing expression grammars always either succeed or fail, and the resulting tree is unique given the grammar and input. In the PVS implementation, we define the trueToGrammar and trueToInput functions and prove the uniqueness results.

\section{A Reference Parser Interpreter}

Now that we have a well-defined notion of computational path, along with well-formed grammars, we can define a parser generator function that is surprisingly simple.

\footnotetext{
${ }^{9}$ It is also possible to put less information into the type system and prove instead lemmas, but such proofs would need to be done by induction, covering all the possible cases each time. When using the type system, the corresponding induction cases are actually split into the type-check conditions. ${ }^{10}$ Here, we are not considering the full set of the nonterminals, but only a grammar node. If this node uses nonterminals, the check of the tree recursively goes into those nonterminals. This notion thus depends on a given set of nonterminals $P_{\exp }$

${ }^{11}$ The full code of the functions trueToGrammar and trueToInput is not given here, at it is only very simple recursive checks. Basically, we do a case analysis on the constructors of the pre_ast datatype. The code can be seen on the github page.
} 


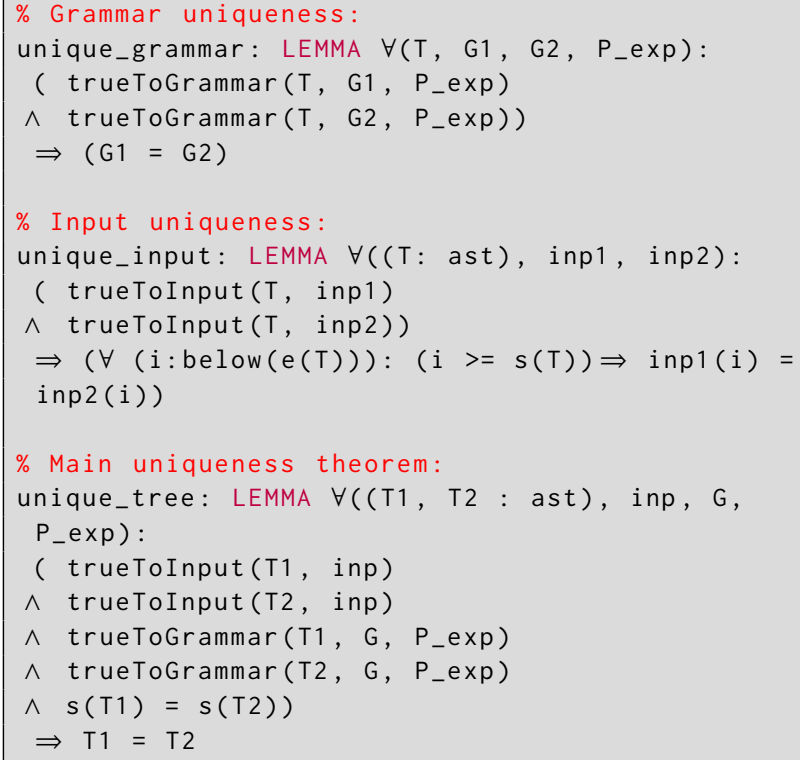

Figure 5. PVS implementation of the uniqueness results.

\subsection{Parser Interpreter}

The peg_parser function is thought to be an interpreter for parsers of any PEG. It takes as input arguments :

- $P_{\text {exp }}$ the interpretation of nonterminals. It must be well-formed.

- $A \in V_{N}$ the current nonterminal.

- $G \sqsubseteq P_{\text {exp }}(A)$ the current grammar node.

- inp the input string, represented as an array of characters

- $b$ the bound of the parsing, less or equal to the length of the input

- $s$ the starting index for the current node

- $s_{T}$ the starting index when the parsing of the current nonterminal started. We ask for $s_{T} \geq s$ and if $s=s_{T}$ (which means nothing was consumed since the start of the current nonterminal), we must have $G$ pattern wellformed. Indeed, as we saw, we allow subexpressions of a given node $P_{\exp }(A)$ to only be structurally wellformed only after consumption of a character.

The output type captures a lot of the complexity of the parsing steps, ensuring mostly that trees consumed or failed coherently with the grammar. The output type is the subset of well-formed trees $T$ that verifies the following conditions. (the implementation is given in figure 6). The three last conditions ensure that the actual result of the parsing corresponds to a property of the grammar.

- $s(T)=s$

- if $\mathrm{G}$ is a star, then $T$ must be a star tree.

- $T \notin \mathcal{S}$ : T is not a skip.

- $T$ is true to the grammar $G$
- $T$ is true to the input $I$

- $(\eta(T)=\top \wedge e(T)=s(T)) \Rightarrow P_{0}(G)$

- $(\eta(T)=\top \wedge e(T)>s(T)) \Rightarrow P_{>0}(G)$

- $(\eta(T)=\perp) \Rightarrow P_{\perp}(G)$

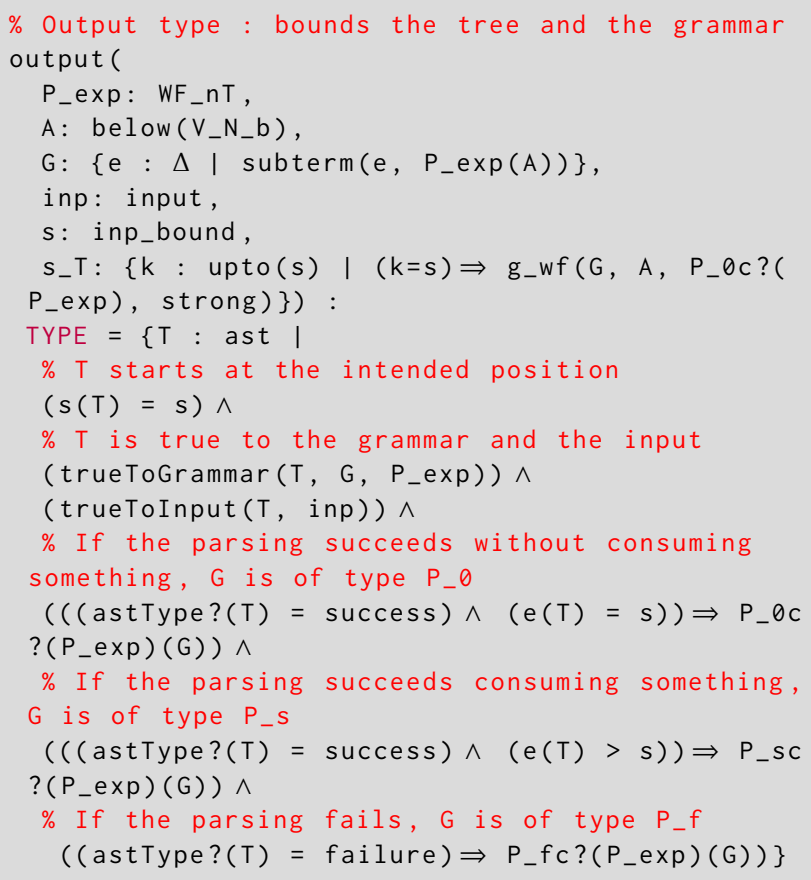

Figure 6. Output type definition. The output function produces a type based on its arguments. The type is expressed by comprehension over the ast type of well-formed grammars.

Those properties ensure that at every step of the computation, the properties needed for termination are preserved. Thanks to all the previous work on grammars and Ast, it is surprisingly simple (see Figure 7). The types contains most of the valuable information.

\subsection{Termination}

The termination is proved by using a strictly decreasing lexicographic order on the 4-tuple :

$$
\left(b-s_{T}, b-s, A,|G|\right)
$$

At each step, we either :

1. Go down the grammar, thus $|G|$ decreases

2. Use a strictly lower nonterminal, thus $A$ decreases

3. In the case of a star in the grammar, both the grammar node, the current nonterminal and $s_{T}$ stay the same, but $s$ increases (because if $e *$ is well-formed, we have $\left.\neg P_{0}(e)\right)$.

4. If we get to a nonTerminal node, and that nonTerminal is greater than the current one, the recursive call is made with $s \leftarrow s_{T}$. Here $s$ must be strictly greater than 


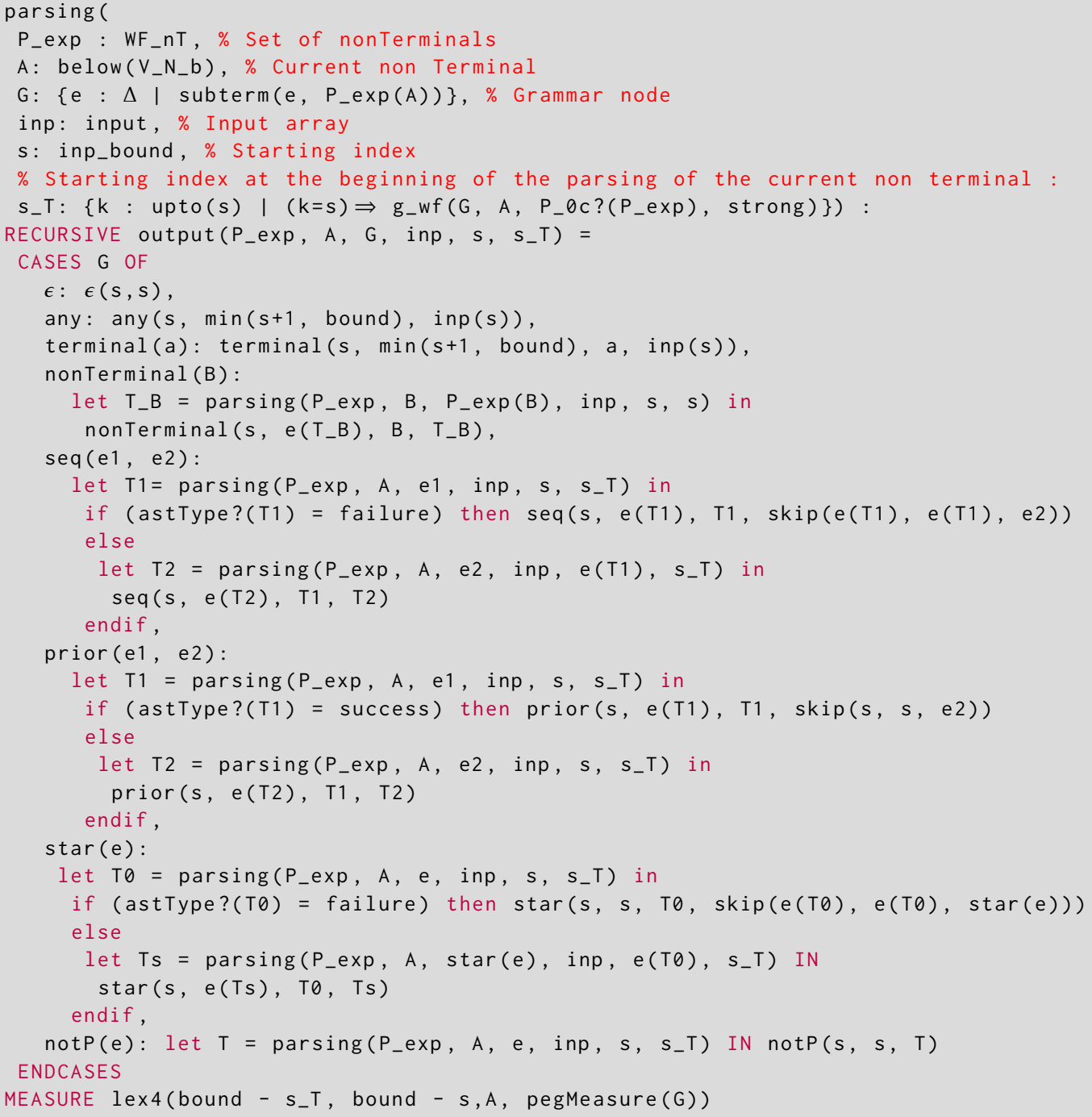

Figure 7. Code for the parser interpreter. The CASES... OF syntax is used to do a case analysis on the possible constructors for the PEG datatype. As for every recursive function in PVS, we provide the measure ensuring termination (lex4 stands for lexical ordering of 4 elements).

$s_{T}$, ensuring that at least one token was consumed before using greater nonTerminal).

\subsection{Satisfaction of the Parsing Rules}

As we mentioned, this reference parser satisfies the rules defined by [11] by design. This was ensured by structure of well-formed ast-trees, but we can prove it a posteriori. It is almost always trivial, as expanding the definition of the parsing functions is enough to show the property. An example is shown in Figure 8. In the actual implementation, the full set of rules is proven.

\subsection{Completeness and Soundness}

As we can see in the output type, the produced trees are true to the input and to the grammar. Thanks to the uniqueness results, this ensures that the only proof of parse or proof of failure is that exists for such a pair of input and grammar is the one found by the parser.

\section{A Packrat Parser Generator}

The natural extension of a PEG parser is to build a packrat parser (see [10]) upon the reference parser. As we already have a reference parser, it is easy to build an efficient packrat parser that only relies on the reference one (through the typing system). The output tree can also be compacted on the fly, removing useless failing branches, while ensuring 


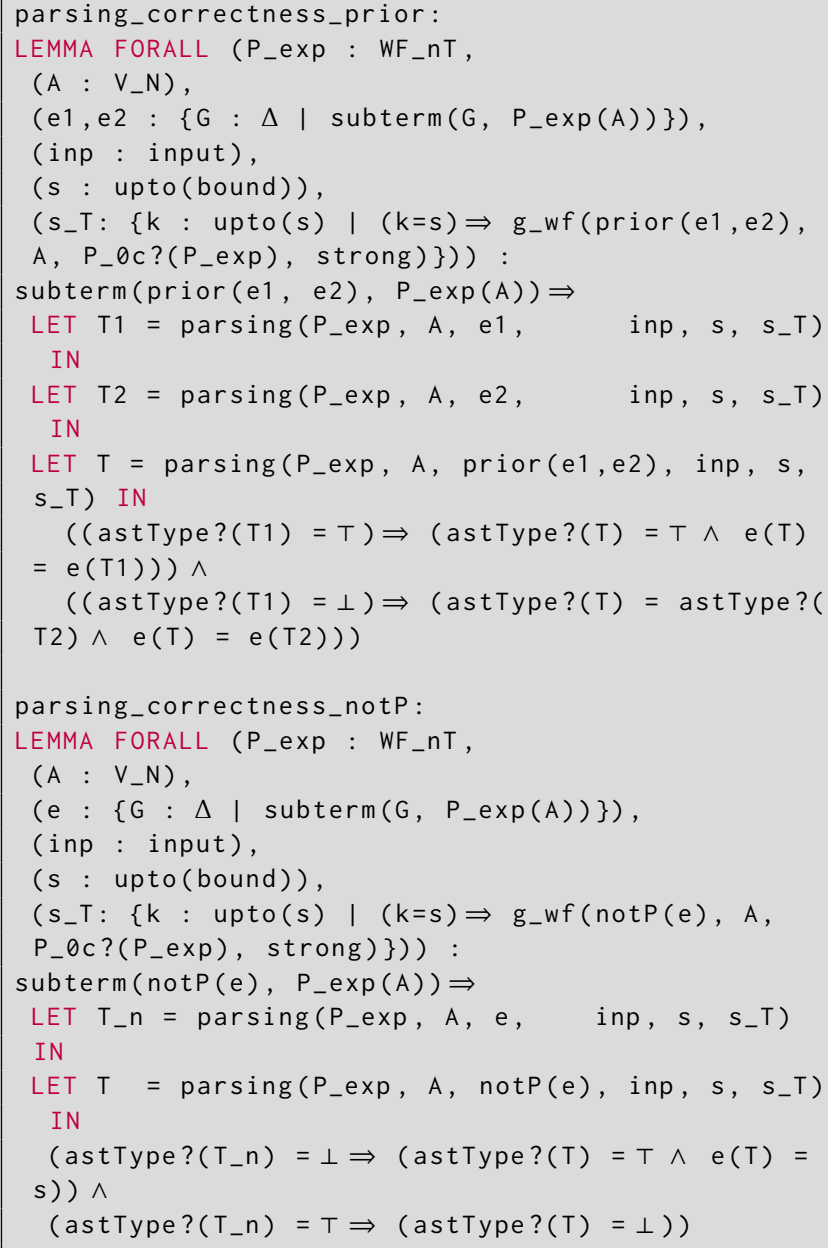

Figure 8. Example of lemmas that shows that the parser verifies the axioms of parsing. In the PVS implementation, the full set of axioms is proven.

that the output is equivalent to the one of the reference parser. This allows us to use the reference parser as a ghost reference while never actually calling it. This whole section is directly inspired by the work of Ford [10].

\subsection{Complexity}

The key to transform the worst case exponential complexity of the parsing into a linear one comes from the following observations:

- Searching for a pattern $A$ at a starting position $s$ is independent from previously done parsing, it only relies on $A$ and $s$.

- There are at most $n$ patterns we can search at most $b$ starting positions

We deduce that if we store the result of the parsing of nonterminals at given starting positions when we compute them (laziness), the parser will be called at most $b \times n$ times. As a recursive call is made in constant time ${ }^{12}$, we end up with a complexity bounded by $O(b \times n)$.

\subsection{Memoization}

We use a PVS structure to store results as they are computed, as shown in Figure 9. The type of those objects is based on the reference parser, ensuring that we only store results that we could obtain by calling the parser with the same parameters. We then modify the parsing function to return both the ast and the record of computed results. When we parse a nonterminal, the result is either already known (and directly returned), or we compute it and update the record of results. This modification is shown in Figure 10.

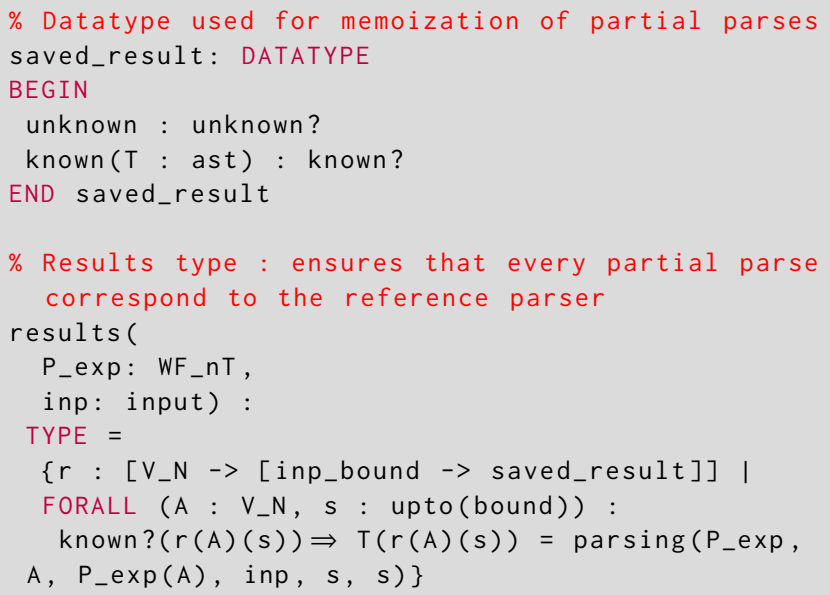

Figure 9. The result structure to store intermediate results as they are computed. A datatype with two constructors is used. The results function produces a type by comprehension over the set of functions which take a nonterminal and a starting index and returns a stored result. Such a stored result should be equal to the reference parser called on the same arguments.

\subsection{Change of the Output Type}

As we mentioned, the reference parser can be used as a reference, stating that the results of the packrat parser are the same as if the reference parser was called with the same arguments. This is illustrated in Figure 11. Again, those conditions are typing conditions, which means that once they are proved through the typechecking condition system, they do not impact the actual computation.

\footnotetext{
${ }^{12}$ In the reference parser implementation, the use of astType function makes it non-constant, but this can be easily changed by modifying the parser so it also outputs the type of the return tree. This is done in the compacted semantic trees of section 6 , as failure trees are reduced to failure nodes, hence making typechecking of trees trivial.
} 


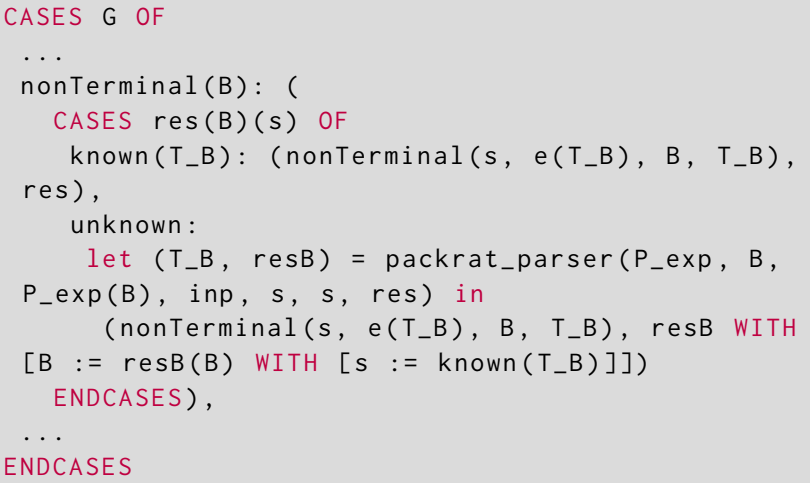

Figure 10. Parsing a nonterminal, checking if the result is already known. If the result is unknown, we compute it and extend the function using the WITH $[\ldots:=\ldots]$ syntax.

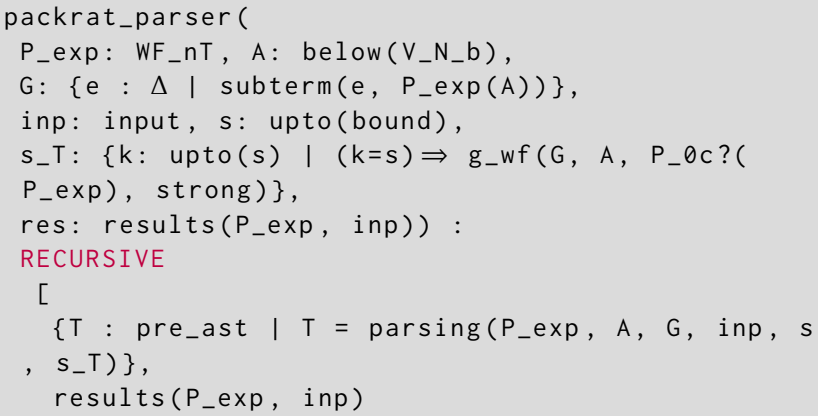

Figure 11. The packrat parser returns a tree that is the same as the reference parser, and a function that satisfies the result type.

\section{Semantic Compacted Tree}

In this section we introduce a modification of the parser that allows the user to specify the data-structure of the parsed result according to their specific needs. Indeed, the AST we defined are interesting as effective proofs of parse ${ }^{13}$ but in practice, users are more interested in data-structures specific to their grammar and not to the underlying PEG operators. For example, an HTML parser should output a DOM tree and not the cumbersome full AST tree of the parser generator. In order to modify the parser to be able to create those semantic trees we need to introduce modifications to the AST type, specify how the user provides the constructors for their data structure, and how we maintain the equivalence with the reference parser.

\footnotetext{
${ }^{13}$ Especially since they provide a complete description of the computational path of the parser.
}

\subsection{Semantic/Failure Node}

To allow the user to produce custom data-structures, we need to introduce a new type for those data-structures : $V_{S}$. In order to simplify the definitions, we do not distinguish between the subtypes the user may be using in the data structure. We only consider that $V_{S}$ is the superset of all user types. We call elements of $V_{S}$ semantic values. When designing a grammar, the user creates nonterminals for each pattern in the intended input. Therefore, the meaningful unit that can be transformed into a semantic value is the result of the parsing of a nonterminal. To replace nonterminal ASTs by their semantic value, we need to add a new constructor to the AST type to store such values. This is shown in Figure 12. Following the same idea, we also introduce a new failure node to replace failing trees that are useless to the user.

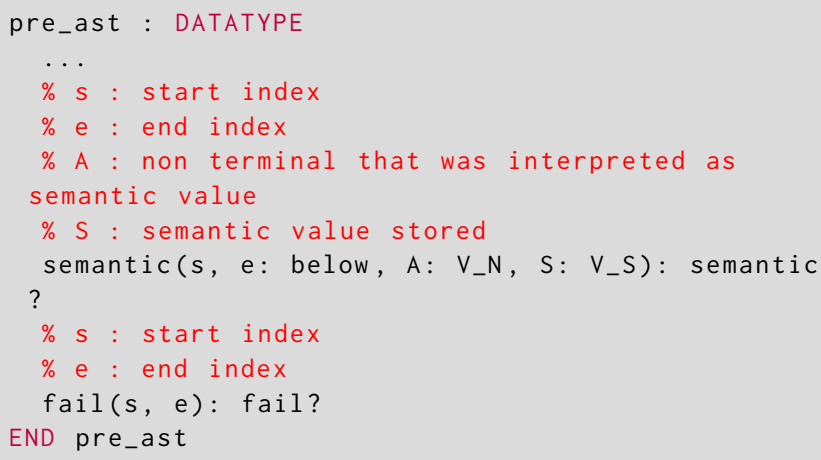

Figure 12. New constructors for the AST type. Those two new constructors are used to compact the tree.

\subsection{Semantic Interpretation of an AST}

Now that we can store semantic values (that correspond to nonterminal subtrees), we can replace those trees by their computed semantic value. This transformation is called the semantic interpretation of a tree. It can be done on a fully computed AST produced by the parser, or on the fly while parsing ${ }^{14}$. The user provides a function that does the transformation of an nonterminal node $\left(P_{\text {inp }}: A S T \longrightarrow V_{S}\right)$ and we do a depth-first search in the tree to recursively replace those nodes by their semantic value. To compact the tree, the semantic interpretation also replaces failing tree by a simple fail node. The implementation is given in Figure $13 .{ }^{15} \mathrm{~A}$ tree that has no nonterminal nodes (but semantic ones) and no failing branches (except for fail nodes) is called a semantic compacted tree. A semantic parser will simply compute the

\footnotetext{
${ }^{14}$ Even though the function can compute semantic interpretations on full trees, the idea is to do it on the fly, and use the function on full tree only to prove equivalence with the reference parser. At runtime, in order to store the smallest possible trees (that can get very big for complex grammars), we compact subtrees as soon as possible by taking their semantic interpretations ${ }^{15} \mathrm{~A}$ few conditions are added to the output type for the sake of simplification later on in the parser.
} 


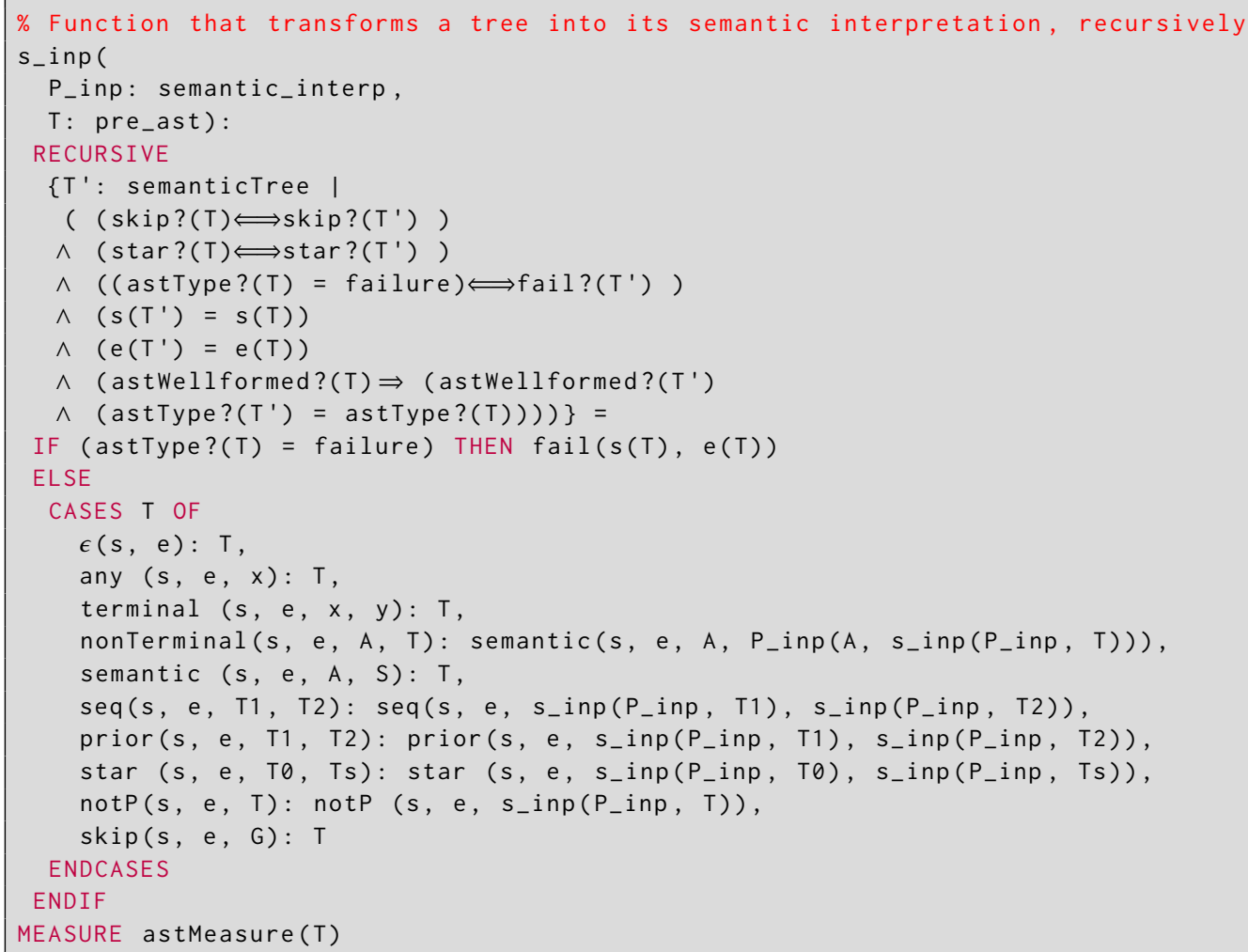

Figure 13. Recursive semantic interpretation of a tree.

semantic interpretation of the nonterminal on the fly during the parsing. A representation of the semantic interpretation of a simple arithmetic expression is given in Figure 14.

\subsection{Semantic Equivalence with Reference Parser AST}

The notion of equivalence with the reference parser becomes more subtle here, as compacted semantic trees are not equal to the trees produced by the reference parser. The main idea is the following: we want to ensure that when information is condensed in a compacted tree, we get the same information if we call the reference parser on the same input and then compact the resulting tree. Here is the detailed definition of equivalence:

1. For all nodes that are not fail and semantic nodes, the reference and compacted trees should be the same.

2. For a fail(s, e) node, we need to check that if we call the reference parser on the same starting point, we would get a failing tree ending on the same index e.

3. For a semantic(s, e, A, S) node, we need to check that the semantic interpretation of the output of the reference parser would be equal to the value stored here.

These conditions on the output of the semantic parser interpreter can be captured directly in the typing system. The output type of a semantic parser interpreter is the following (using the s_inp function).
$\left\{T\right.$ : pre_ast $\mid T=s_{-} i n p\left(P_{-} i n p\right.$, parsing $\left(P_{-} \exp , A\right.$, $G$, inp, b, s, s_T)) \}

Example. The PVS suite provides an interactive interface to execute the verified code: PVSio [9]. Using this system, we can test the parser on real examples. We crafted a very simple arithmetic expression parser for test purposes: the expression is represented as an array of ascii values $(1+2 *(3-4 / 5)$ is represented as the sequence $[49,43,50,42,40,51,45,52,47,53,41]$ with binary values). The interaction with the system is shown in Figure 15. We can see that the semantic parser does produce a compacted tree that correspond to the reference one, and that modifying the input changes the result accordingly.

\section{Conclusions}

Extracting data from input streams representing programs, text, documents, images, and video is a complex task. Parsers for these data formats transform the input data streams into actionable data while rejecting incorrect inputs. Parsing is supported by a rich body of theory, but it is also central to practice. Many software vulnerabilities arise from poorly designed grammars, ambiguous inputs, and incorrect parsing. Parsing expression grammars are a widely used class of 


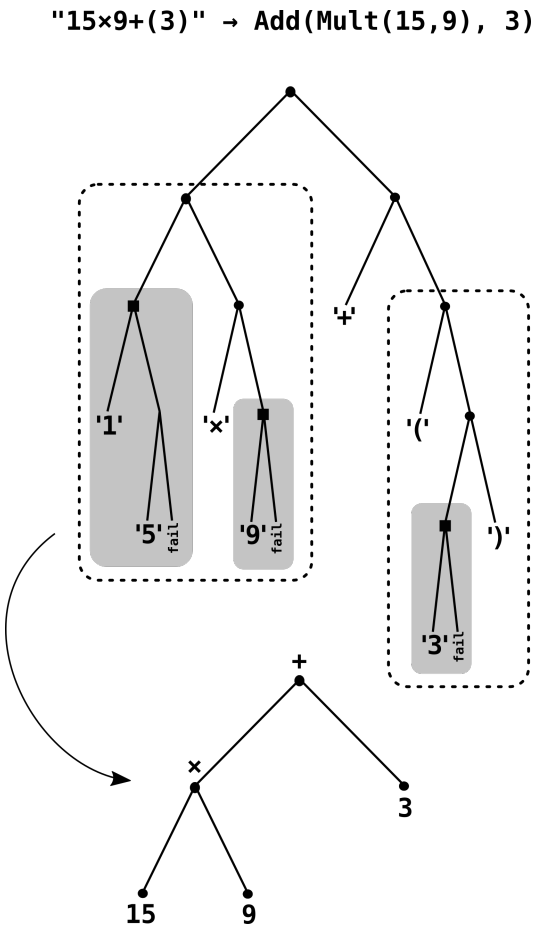

Figure 14. Representation of the semantic interpretation of simple arithmetic expression. Numbers are parsed as lists of digits (grey boxes).

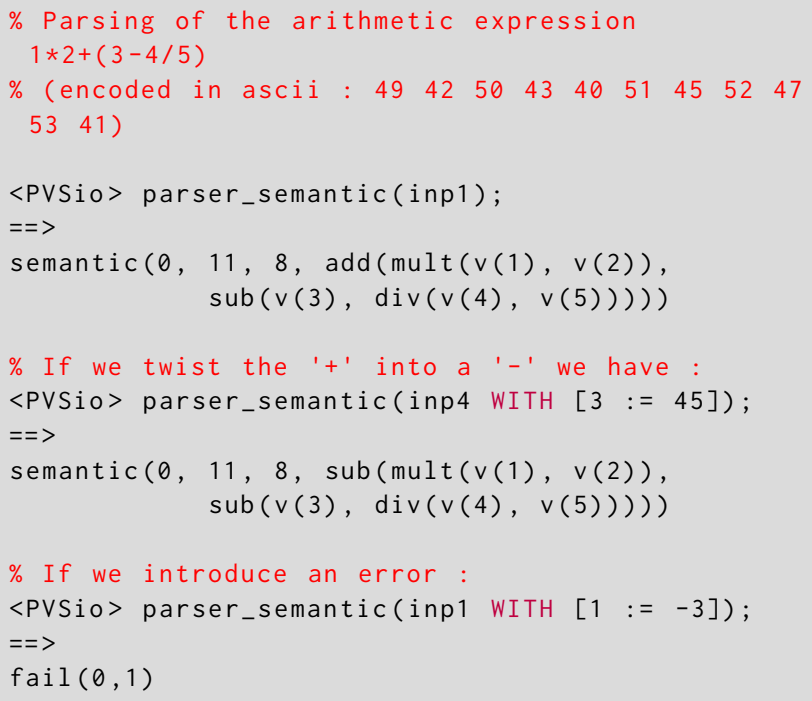

Figure 15. Example of using the semantic parser for simple arithmetic expressions

expressive grammars that support efficient and unambiguous packrat parsing through memoization. We have used PVS to formalize the metatheory of PEGs and derived a correct parser interpreters for these grammars supporting memoization and semantic actions. The proofs have been mechanically verified in PVS by taking advantage of the expressiveness of the PVS type system. A significant part of the metatheory covers the analysis of grammars constructed from PEG expressions to check if a parse based on the expression could fail, could succeed without consuming input, or could succeed only by consuming input. This analysis is used to establish the termination of a reference parser interpreter for PEGs. We also define a parse tree representation that captures the trace of the parser on an input. This representation serves as a evidence for the parsing of the input with respect to a given grammar. We establish certain uniqueness results that demonstrate that there is exactly one grammar with respect to parse tree for a given input, and exactly one parse tree for a given input with respect to a given grammar. These uniqueness results demonstrate that the reference parser is complete: it either returns a successful parse tree and there is a unique such tree, or a failed parse tree and there is no other parse tree that parses the same input with success or failure.

In future work, we plan to conduct empirical studies of the performance of the parsers, explore the use of efficient data structures for parsing, develop a systematic methodology for the derivation of correct-by-construction parser interpreters and generators for other grammar formats, and experiment with the integration of the generated parsers into security-critical applications. Our work is a preliminary step toward powerful correct-by-construction parser interpreters and generators for expressive grammars and data format descriptions.

\section{References}

[1] Alfred V. Aho, Ravi Sethi, and Jeffrey D. Ullman. 1986. Compilers: Principles, Techniques, and Tools. Addison-Wesley.

[2] Aditi Barthwal and Michael Norrish. 2009. Verified, executable parsing. In Programming Languages and Systems, 18th European Symposium on Programming, ESOP 2009 (Lecture Notes in Computer Science), Giuseppe Castagna (Ed.), Vol. 5502. Springer, 160-174.

[3] Jean-Philippe Bernardy and Patrik Jansson. 2016. Certified contextfree parsing: A formalisation of Valiant's algorithm in Agda. Logical Methods in Computer Science 12, 2 (2016), 1-28. https://doi.org/10.2168/ LMCS-12(2:6)2016

[4] Ana Bove, Peter Dybjer, and Ulf Norell. 2009. A brief overview of Agda - A functional language with dependent types. In Theorem Proving in Higher Order Logics, 22nd International Conference, TPHOLs 2009, Munich, Germany, August 17-20, 2009. Proceedings (Lecture Notes in Computer Science), Stefan Berghofer, Tobias Nipkow, Christian Urban, and Makarius Wenzel (Eds.), Vol. 5674. Springer, 73-78. https://doi. org/10.1007/978-3-642-03359-9_6

[5] Edwin Brady. 2013. Idris, a general-purpose dependently typed programming language: Design and implementation. F. Funct. Program. 23, 5 (2013), 552-593. https://doi.org/10.1017/S095679681300018X

[6] Sergey Bratus, Lars Hermerschmidt, Sven M. Hallberg, Michael E. Locasto, Falcon Momot, Meredith L. Patterson, and Anna Shubina. 2017. Curing the vulnerable parser: Design patterns for secure input handling. ;login: 42, 1 (2017), 33-39.

[7] William H. Burge. 1975. Recursive Programming Techniques. AddisonWesley, Reading, MA. 
[8] Benjamin Delaware, Sorawit Suriyakarn, Clément Pit-Claudel, Qianchuan Ye, and Adam Chlipala. 2019. Narcissus: Correct-byconstruction derivation of decoders and encoders from binary formats. PACMPL 3, ICFP (2019), 82:1-82:29. https://doi.org/10.1145/3341686

[9] Aaron Dutle, César A. Muñoz, Anthony Narkawicz, and Ricky W. Butler. 2015. Software validation via model animation. In Tests and Proofs - 9th International Conference, TAP 2015, Held as Part of STAF 2015, L'Aquila, Italy, fuly 22-24, 2015. Proceedings (Lecture Notes in Computer Science), Jasmin Christian Blanchette and Nikolai Kosmatov (Eds.), Vol. 9154. Springer, 92-108. https://doi.org/10.1007/978-3-31921215-9_6

[10] Bryan Ford. 2002. Packrat parsing: : Simple, powerful, lazy, linear time, functional pearl. In Proceedings of the Seventh ACM SIGPLAN International Conference on Functional Programming (ICFP '02), Pittsburgh, Pennsylvania, USA, October 4-6, 2002., Mitchell Wand and Simon L. Peyton Jones (Eds.). ACM, New York, NY, USA, 36-47. https://doi.org/10.1145/581478.581483

[11] Bryan Ford. 2004. Parsing expression grammars: A recognition-based syntactic foundation. In Proceedings of the 31st ACM SIGPLAN-SIGACT Symposium on Principles of Programming Languages, POPL 2004, Venice, Italy, January 14-16, 2004, Neil D. Jones and Xavier Leroy (Eds.). ACM, 111-122. https://doi.org/10.1145/964001.964011

[12] Richard A. Frost and John Launchbury. 1989. Constructing natural language interpreters in a lazy functional language. Comput. f. 32, 2 (1989), 108-121. https://doi.org/10.1093/comjnl/32.2.108

[13] Graham Hutton. 1989. Parsing Using combinators. In Functional Programming, Proceedings of the 1989 Glasgow Workshop, 21-23 August 1989, Fraserburgh, Scotland, UK (Workshops in Computing), Kei Davis and John Hughes (Eds.). Springer, 353-370.

[14] Jacques-Henri Jourdan, François Pottier, and Xavier Leroy. 2012. Validating LR(1) parsers. In Programming Languages and Systems - 21st European Symposium on Programming, ESOP 2012, Held as Part of the European foint Conferences on Theory and Practice of Software, ETAPS 2012, Tallinn, Estonia, March 24 - April 1, 2012. Proceedings (Lecture Notes in Computer Science), Helmut Seidl (Ed.), Vol. 7211. Springer, 397-416.

[15] Adam Koprowski and Henri Binsztok. 2011. TRX: A formally verified parser interpreter. Logical Methods in Computer Science 7, 2 (2011), 1-26. https://doi.org/10.2168/LMCS-7(2:18)2011

[16] Ramana Kumar, Magnus O. Myreen, Michael Norrish, and Scott Owens. 2014. CakeML: A verified implementation of ML. In The 41st Annual ACM SIGPLAN-SIGACT Symposium on Principles of Programming Languages, POPL '14, San Diego, CA, USA, fanuary 20-21, 2014, Suresh Jagannathan and Peter Sewell (Eds.). ACM, 179-192. https://doi.org/10.1145/2535838.2535841

[17] Sam Lasser, Chris Casinghino, Kathleen Fisher, and Cody Roux. 2019. A verified LL(1) parser generator. In 10th International Conference on Interactive Theorem Proving, ITP 2019, September 9-12, 2019, Portland, OR, USA. (LIPIcs), John Harrison, John O'Leary, and Andrew Tolmach (Eds.), Vol. 141. Schloss Dagstuhl - Leibniz-Zentrum für Informatik, 24:1-24:18. https://doi.org/10.4230/LIPIcs.ITP.2019.24
[18] Xavier Leroy. 2009. Formal verification of a realistic compiler. Commun. ACM 52, 7 (2009), 107-115. http://doi.acm.org/10.1145/1538788. 1538814

[19] Raul Lopes, Rodrigo Geraldo Ribeiro, and Carlos Camarão. 2016. Certified derivative-based parsing of regular expressions. In Programming Languages - 20th Brazilian Symposium, SBLP 2016, Maringá, Brazil, September 22-23, 2016, Proceedings (Lecture Notes in Computer Science), Fernando Castor and Yu David Liu (Eds.), Vol. 9889. Springer, 95-109. https://doi.org/10.1007/978-3-319-45279-1_7

[20] Greg Morrisett, Gang Tan, Joseph Tassarotti, Jean-Baptiste Tristan, and Edward Gan. 2012. RockSalt: Better, faster, stronger SFI for the x86. In ACM SIGPLAN Conference on Programming Language Design and Implementation, PLDI '12, Beijing, China - fune 11 - 16, 2012, Jan Vitek, Haibo Lin, and Frank Tip (Eds.). ACM, 395-404. https://doi.org/ 10.1145/2254064.2254111

[21] Sam Owre, John Rushby, Natarajan Shankar, and Friedrich von Henke. 1995. Formal Verification for Fault-Tolerant Architectures: Prolegomena to the Design of PVS. 21, 2 (Feb. 1995), 107-125. PVS home page: http://pvs.csl.sri.com.

[22] Tahina Ramananandro, Antoine Delignat-Lavaud, Cédric Fournet, Nikhil Swamy, Tej Chajed, Nadim Kobeissi, and Jonathan Protzenko. 2019. EverParse: Verified secure zero-copy parsers for authenticated message formats. In 28th USENIX Security Symposium, USENIX Security 2019, Santa Clara, CA, USA, August 14-16, 2019, Nadia Heninger and Patrick Traynor (Eds.). USENIX Association, 1465-1482. https://www.usenix.org/conference/usenixsecurity19/ presentation/delignat-lavaud

[23] Tom Ridge. 2011. Simple, Functional, Sound and Complete Parsing for All Context-Free Grammars. In Certified Programs and Proofs First International Conference, CPP 2011, Kenting, Taiwan, December 7-9, 2011. Proceedings (Lecture Notes in Computer Science), Jean-Pierre Jouannaud and Zhong Shao (Eds.), Vol. 7086. Springer, 103-118.

[24] Gang Tan and Greg Morrisett. 2018. Bidirectional grammars for machine-code decoding and encoding. f. Autom. Reasoning 60, 3 (2018), 257-277. https://doi.org/10.1007/s10817-017-9429-1

[25] Mark Tullsen, Lee Pike, Nathan Collins, and Aaron Tomb. 2018. Formal verification of a Vehicle-to-Vehicle (V2V) messaging system. In Computer Aided Verification - 30th International Conference, CAV 2018, Held as Part of the Federated Logic Conference, FloC 2018, Oxford, UK, Fuly 14-17, 2018, Proceedings, Part II (Lecture Notes in Computer Science), Hana Chockler and Georg Weissenbacher (Eds.), Vol. 10982. Springer, 413-429. https://doi.org/10.1007/978-3-319-96142-2_25

[26] Marcell van Geest and Wouter Swierstra. 2017. Generic packet descriptions: verified parsing and pretty printing of low-level data. In Proceedings of the 2nd ACM SIGPLAN International Workshop on Type-Driven Development, TyDe@ICFP 2017, Oxford, UK, September 3, 2017, Sam Lindley and Brent A. Yorgey (Eds.). ACM, 30-40. https: //doi.org/10.1145/3122975.3122979

[27] Qianchuan Ye and Benjamin Delaware. 2019. A verified protocol buffer compiler. In Proceedings of the 8th ACM SIGPLAN International Conference on Certified Programs and Proofs, CPP 2019, Cascais, Portugal, Fanuary 14-15, 2019, Assia Mahboubi and Magnus O. Myreen (Eds.). ACM, 222-233. https://doi.org/10.1145/3293880.3294105 